\title{
Nanostructure and Magnetic Properties of FeZr/CuZr Amorphous Multilayers
}

\author{
Ming SUN ${ }^{a, *}$,Tao FENG ${ }^{b}$, Guo PENG, Abdur RAUF and Bin WU \\ Herbert Gleiter Institute of nanoscience, Nanjing university of Science and Technology 210094 \\ Nanjing, China \\ a32600473@qq.com \\ btao.feng@njust.edu.cn
}

Keywords: Multilayers, Amorphous, Magnetic property.

\begin{abstract}
Fe}_{75} \mathrm{Zr}_{25} / \mathrm{Cu}_{64} \mathrm{Zr}_{36}$ multilayers metallic glass thin films have been prepared by magnetron sputtering herein. The magnetic studies shown that the saturation magnetization of this alloys increase after annealing under glass transition temperature $\left(\mathrm{T}_{\mathrm{g}}\right)$. The change of the magnetization might be attribute to structural transformation triggered by diffusion. This work offers an avenue to modify the nanostructure of thin films, and further to manipulate the physical properties of metallic glasses.
\end{abstract}

\section{Introduction}

Metallic glasses (MGs), or amorphous metallic alloys, are the form of noncrystalline materials with absence of long-range atomic ordering and/or periodicity as they are generally formed by faster rate melt quenching for stable glassy state retention. MGs are non-equilibrium materials which have emerged as a promising candidate for the novel engineering applications owing to their superior properties such as soft magnetic, excellent mechanical properties (i.e. high specific strength, large elastic limits up to $\sim 2 \%$ ) and high wear and corrosion resistance $[1,2,3,4,5]$. However, MGs have some limitations such as the exhibit poor room temperature ductility $(<2 \%)$ and small product size due to limited glass forming ability [6]. Recently, thin film metallic glasses (TFMGs) with the nanoscale structural morphology have attracted great attentions as they have flexibility in fabricating complicate shape samples and possesses improved mechanical properties (i.e., good ductility and fatigue resistance due to their low dimension, which offers new opportunities to use them in micro- and nanoscale electronic devices [1].Basically the reduced granular size directly effects the volume fraction of atoms located at the glass-glass interfaces which plays an important role in determining the performance of these TFMGs [2]. Similar to nanocrystalline materials, varying the granule size of the MNGs thereby modifies their properties.

Multilayers thin films consist of alternate layers elements/alloys deposited on various substrates. Such type of multilayers nanostructures have many valuable applications (e.g. magnetic sensors in $\mathrm{read} / \mathrm{write}$ heads and in memory devices).Interface is a common unit which exists in multilayers and it has played a very important role in the manipulating the structure related properties of material $[7,8]$. It is a well-known fact that any changes in the interfacial region between two layers in such nanostructures can greatly influence their physical properties [9]. The typical interface width in most of the multilayers ranges in the order of a few nanometer. Therefore; in this context, nanoscale diffusion across the interfaces have great influence on modifying their properties, thus it has acquired a great significance. In general, a system usually seeking for a lowest Gibbs free energy state and this state of energy can be achieved through atomic diffusion for which the presence of a driving force (e.g. a chemical driving force) is needed.

Diffusion mechanisms in amorphous metallic alloys are not well understood. This is partly due to the difficulty in measuring the very small diffusion coefficients which are typical of metallic glasses below their crystallization temperatures. Understanding the diffusion in metallic glasses can demonstrate the theory of solid state reactions[10]. Furthermore; self-diffusion of the constituent 
species in a thin film may also occur which is equally important. Such a diffusion is responsible for the structural transformations which ultimately affect all the physical properties of thin films [11].

We choose two amorphous systems, $\mathrm{Fe}_{75} \mathrm{Zr}_{25}$ and $\mathrm{Cu}_{64} \mathrm{Zr}_{36}$, which show high glass forming ability to construct metallic glass multilayers. $\Delta \mathrm{H}$ for $\mathrm{Fe}-\mathrm{Zr}$ is $-25 \mathrm{~kJ} / \mathrm{mol}$ and $\Delta \mathrm{H}$ for $\mathrm{Cu}-\mathrm{Zr}$ is $23 \mathrm{~kJ} / \mathrm{mol}$, but $\Delta \mathrm{H}$ for $\mathrm{Fe}-\mathrm{Cu}$ is $13 \mathrm{~kJ} / \mathrm{mol}$ [12] $\mathrm{Fe}$ and $\mathrm{Cu}$ are commonly considered immiscible elements. In our system of $\mathrm{FeZr} / \mathrm{CuZr}$ multilayers, $\mathrm{Zr}$ is the common constituent element in both of the layers, therefore; its interfacial diffusion or self-diffusion must be determined. Usually, the diffusion rely on the diffusion activation energy, which is related with chemical composition, structure, size of the atoms, etc. If it is possible to control the process of diffusion, then we can control the region of interface, and further manipulate the interface to realize the different properties (magnetic and mechanical properties). Chen et al[2] prepared different component of $\mathrm{Fe}_{90} \mathrm{Sc}_{10}$ and $\mathrm{Cu}_{64} \mathrm{Sc}_{36}$ system, with effect of the interface, the magnetization of the $\left(\mathrm{Fe}_{90} \mathrm{Sc}_{10}\right)_{0.74}\left(\mathrm{Cu}_{64} \mathrm{Sc}_{36}\right)_{0.26}$ alloy is even better than the pure $\mathrm{Fe}_{90} \mathrm{Sc}_{10}$ metallic nanoglass. In Martina Ahlberg' work[13] the interface have made critical temperature anomalous enhance when the thickness of the magnetic layer is decreased from $6 \mathrm{~nm}$ to $2 \mathrm{~nm}$ in the amorphous FeZr/AlZr multilayers. It will be anticipated for us to achieve some extraordinary properties with manipulating the interface.

\section{Experimental Procedure}

The multilayer thin films were deposited by using JGP650 dual chamber magnetron sputtering facility, which is equipped with computer controlled two separate vacuum chambers i.e. sample loading chamber and sputtering unit, in order to avoid any contamination and ensure high vacuum stability. All the sample were deposited on $\mathrm{Si}$ (111) substrates at room temperature. Before deposition, the chamber was pumped down with a turbo-molecular pump system allowing a base vacuum of $\sim 10^{-5} \mathrm{~Pa}$ before refilling with argon at the desired pressure around $\sim 0.5 \mathrm{~Pa}$. we used two separate alloys targets, $F_{75} Z_{25}$ (diameter $\Phi=50 \mathrm{~mm}$, thickness=3mm), and $\mathrm{Cu}_{64} \mathrm{Zr}_{36}$ targets $(\Phi=50 \mathrm{~mm}$, thickness $=3 \mathrm{~mm})$, this composition was reported with high glass-forming ability $[14,15]$. $30 \mathrm{~W}$ Direct current (DC) power was used. The argon flow rate set as $40 \mathrm{sccm}$ for all the experiments. The films growth rate was measured using cross sectional SEM thickness measurement. With computer control systems, we can easily control the shutters and the sputtering time of each targets. With vacuum furnace inside the machine, we can anneal the sample up to threshold glass transition point in high vacuum, to reduce the oxidation.

The crystallographic structure were explored by X-ray diffraction (XRD) using Brucker D8 Advance system using $\mathrm{Cu} \mathrm{K \alpha}$ radiation $(\lambda=0.154 \mathrm{~nm})$. The working voltage of the instrument was $40 \mathrm{kV}$ with a current of $40 \mathrm{~mA}$. The model of SEM is the Quant $250 \mathrm{FEG}$, the magnification is 14 to $1000,000 \mathrm{X}$, and it clearly see the thickness of the film and the morphology. TEM samples were prepared by focused ions Beam (FIB), a powerful tool in the nanoscale and the Cross-Beam System inside combines the 3D imaging and analysis performance of the GEMINI e-Beam column with the ability of a FIB for material processing and sample preparation at nanoscale. The morphological and compositional characterization of samples were done with FEI Tecnai 20, which is the most powerful atomic resolution transmission electron microscope (TEM) with the large acceleration voltage range of 20 to $200 \mathrm{kV}$ for morphological characterization and electron diffraction. The magnetic properties were performed under the physical property measurement system (PPMS).Themagnetic field can up to $9 \mathrm{~T}$ and the temperature can vary from 2 to 400K. Magnetic measurements for this multilayers were conducted under $4 \mathrm{~K}$, with field from $-2 \mathrm{~T}$ to $2 \mathrm{~T}$.

\section{Results and Discussion}

\section{Composition and Structural Characterization}

$\mathrm{Cu}_{64} \mathrm{Zr}_{36}$ and $\mathrm{Fe}_{75} \mathrm{Zr}_{25}$ are usedas primary sources to prepare multilayers. As using alloy targets can effectively control the composition and avoid the oxidation. The multilayers of $\mathrm{Fe}_{75} \mathrm{Zr}_{25}$ and $\mathrm{Cu}_{64} \mathrm{Zr}_{36}$ are proved to be amorphous with XRD. The XRD pattern is shown in Fig.1. 


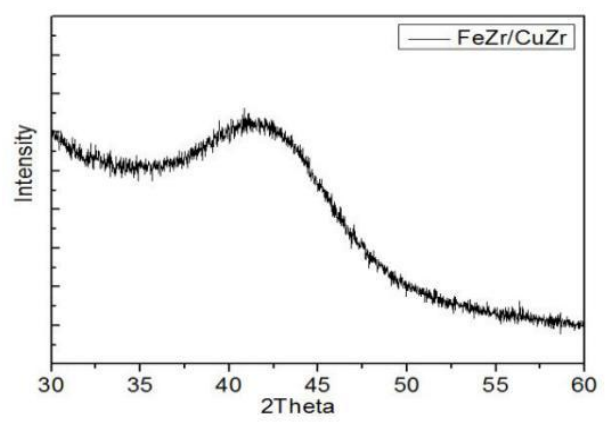

Fig.1.The XRD of $\mathrm{FeZr} / \mathrm{CuZr}$

As shown in SEM picture (Fig. 2a), the surface of the sample has some cracks. This kind of structure can usually prepare by sputtering technique, in which column state are formed. And with cross section of SEM (Fig. 2b), the thickness of the film are measured. From the thickness the growth rate can be calculated easily, thus, it is convenient for us to prepare thin film with particular thickness.
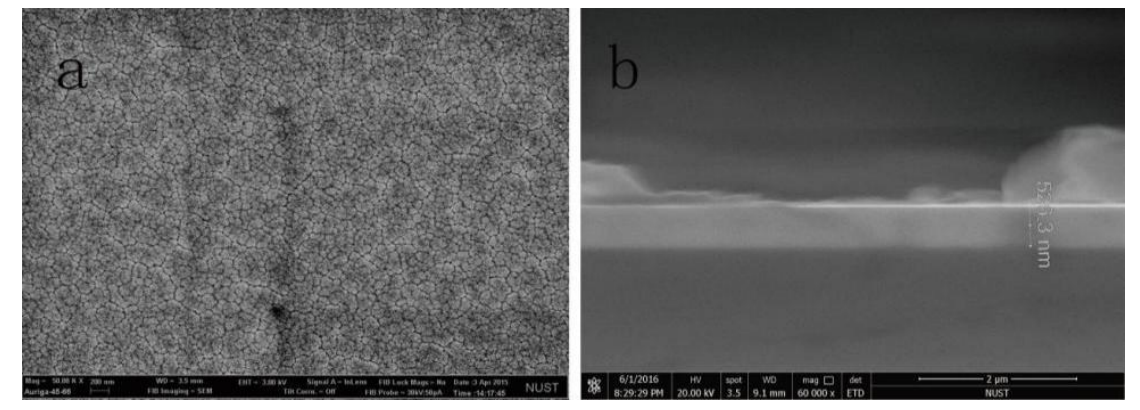

Fig.2 (a)The morphology of Multilayers and, (b) the cross-section of the film.

\section{Microstructure of Multilayers}

Microstructures of multilayers are analyzed by TEM micrographs. The amorphous structure is easily confirmed by HRTEM, as shown in Fig. 3a, the atoms are distributed disordered, no evidence of any crystallites in the morphology. The different contrast in the pattern, it is due to the different atomic number of our composition. The bigger the atomic number, the darker in the picture. And the interface between the two layers is rough. From the inserted selected area electron diffraction (SAED) pattern(Fig. 3b), two diffused rings are presented, they can be corresponding to the two different structures in the multilayers.
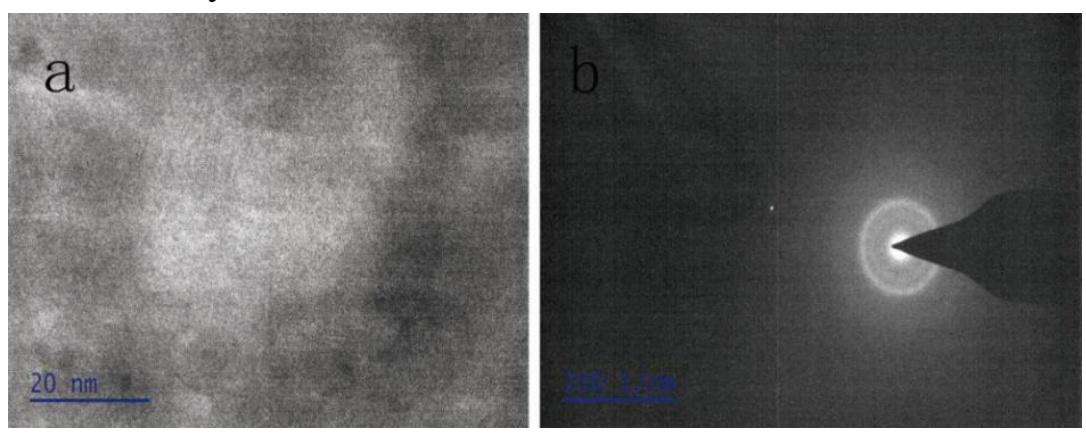

Fig.3 (a) TEM of multilayers and (b)The SAED of multilayers. 


\section{Thermodynamic of Multilayer}

The thermodynamic properties of the sample were measured by DSC from room temperature to $873 \mathrm{~K}$, with $20 \mathrm{~K} / \mathrm{min}$ scan rate. Andit clearly shows the transition from amorphous to crystalline. And the $T_{g}$ can be deduced from the curve, the $T_{g}$ of $\mathrm{CuZr}$ is $733 \mathrm{~K}$, the $\mathrm{T}_{\mathrm{g}}$ of $\mathrm{FeZr}$ is $743 \mathrm{~K}$, as shown in Fig. 4. The results are similar with others. [16,17]

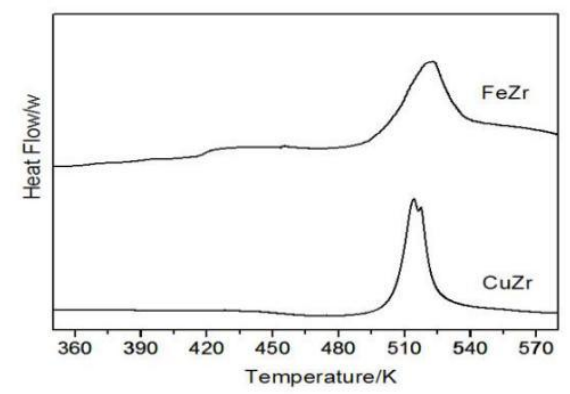

Fig.4.The DSC curves of FeZr and CuZrmetallic glasses.

\section{Magnetic Properties of FeZr/CuZr Multilayers}

The magnetic moment of $\mathrm{FeZr} / \mathrm{CuZr}$ was measured to get the relationship between structure and property. One sample (original sample) is the multilayer without any treatment, the other one (annealing sample) is under annealing in high vacuum in $573 \mathrm{~K}$ (which is lower than $\mathrm{T}_{\mathrm{g}}$ ) for $1 \mathrm{~h}$. The results show that after annealing, the magnetic moment of multilayer show a large increase, from $0.8 \mu_{\mathrm{B}}$ to $1.5 \mu_{\mathrm{B}}$, which is correspondent with our hypothesis that annealing enlarge the diffusion in the multilayers, causing the transformation of the amorphous structure (Fig. 5). So It is meaningful for us that changing the structure of material, we can get the different properties.

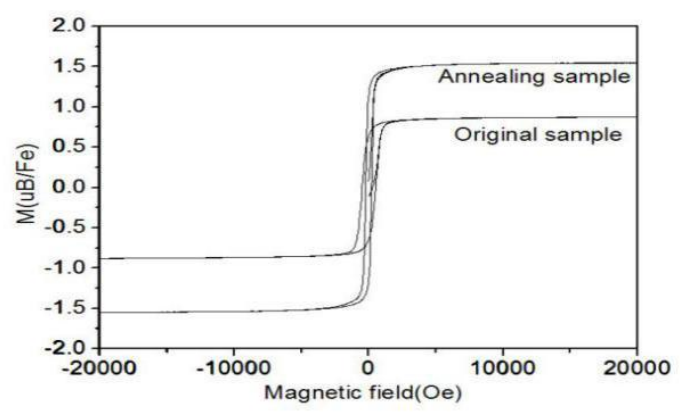

Fig.5. The magnetization plots of $\mathrm{FeZr} / \mathrm{CuZr}$ multilayers before and after annealing.

\section{Conclusions}

Multilayers of amorphous $\mathrm{FeZr} / \mathrm{CuZr}$ have been prepared with sputtering. The SEM results reveal the thickness of the layers and are helpful for us to control the thickness of films. With XRD and TEM, the amorphous structure are easily confirmed.The Tg of the amorphous multilayers can be observed from DSC curve. The magnetic properties of multilayers can be tuned by structural transformation triggered by diffusion. It is obviously that the diffusion and interface effects in the multilayers are very important for materials' unique properties. 


\section{References}

[1] Wahyu Diyatmika, Jinn P. Chu, Berhanu Tulu Kacha, Chia-Chi Yu, Cheng-Min Lee, Thin film metallic glasses in optoelectronic, magnetic, and electronic applications: A recent update, Current Opinion in Solid State and Materials Science19 (2015) 95-106.

[2] Na Chen, Di Wang, Tao Feng, Robert Kruk, Ke-fu Yao, Dmtri V. Louzguine Luzgin, Horst Hahn, and Herbert Gleiter, A nanoglass alloying immiscible $\mathrm{Fe}$ and $\mathrm{Cu}$ at the nanoscale, Nanoscale, 7(15) 2015, 6607-6611.

[3] J.P. Chu, J.S.C. Jang, J.C. Huang, H.S. Chou, Y. Yang, J.C. Ye, Y.C. Wang, J.W. Lee, F.X. Liu, P.K. Liaw, Y.C. Chen, C.M. Lee, C.L. Li, C. Rullyani, Thin film metallic glasses: Unique properties and potential applications, Thin Solid Films 520(2012) 5097-5122.

[4] Zhi Li, Cheng Zhang, Lin Liu, Wear behavior and corrosion properties of Fe-based thin film metallic glasses, Journal of Alloys and Compounds 650 (2015) 127-135.

[5] C. Zhang, R.Q. Guo, Y. Yang, Y. Wu, L. Liu, Influence of the size of spraying powders on the microstructure and corrosion resistance of Fe-based amorphous coating, Electrochimica. Acta 56 (2011) 6380-6388.

[6] G. Kumar, A. Desai, J. Schroers, Bulk metallic glass: the smaller the better, Advanced. Materials. 23 (2011) 461-476.

[7] Z. K. Li, H. M. Fu, P. F. Sha, Z. W. Zhu, A. M. Wang, H. Li, H. W. Zhang, H. F. Zhang \& Z. Q. Hu, Atomic interaction mechanism for designing the interface of W/Zr-based bulk metallic glass composites, Scientific Reports 5, Article number: 8967 (2015).

[8] Omar Adjaoud and Karsten Albe, Interfaces and interphases in nanoglasses: Surface segregation effects and their implications on structural properties, Acta Materialia 113 (2016) 284292.

[9] Kim, W.-S., Andra, W., Kleemann, W. Influence of interfaces on the perpendicular magnetic anisotropy in Tb/Fe multilayers. Physical. Review. B 58, 6346-6352 (1998).

[10]H. Hahn and R.S. Averback. Dependence of tracer diffusion on atomic size in amorphous Ni-Zr. Physical. Review. B 37, 6533-6535 (1988).

[11] Sharma, P., Gupta, A. Effect of preparation condition on the soft magnetic properties of FeCuNbSiB thin films. Journal of Magnetism and Magnetic Materials. 288, 347-353 (2005).

[12] Akira Takeuchi and Akihisa Inoue. Calculations of Mixing Enthalpy and Mismatch Entropy for Ternary Amorphous Alloys. Materials Transaction. 41(11), 1372-1378 (2000)

[13]Martina Ahlberg, Atieh Zamani, Erik Ostman. Reversed interface effects in amorphous FeZr/AlZr multilayers.Physical. Review. B.90(18), 184403(1-5) (2014.

[14] O.J. Kwon, Y. C. Kim, K.B. Kim, Y.K. Lee, and E. Fleury. Formation of Amorphous Phase in the Binary Cu-Zr Alloy System. Metalsand materials international 12(3),207-212(2006)

[15]Debabrata Mishra, A Perumal and A Srinivasan. Magnetic properties of mechanically alloyed Fe100-xZrx $(20 \leq x \leq 35)$ powder. Journal of Physics D: Applied Physics. 41(21),1-8(2008)

[16]B.F. Lu, J.F. Li, L.T. Kong, Y.H. Zhou. Correlation between mechanical behavior and glass forming ability of Zr-Cu metallic glasses.Intermetallics.19(7),1032-1035(2011)

[17]R Pizsrro, J S Garitaonandia,F Plazaola, J M Barandiaran and J M Greneche. Magnetic and Mossbauer study of multiphase $\mathrm{Fe}-\mathrm{Zr}$ amorphous powders obtained by high energy ball milling. Journal of Physics: Condensed Matter.12(13),3101-3112(2000) 
[18]P.T.Korelis,P.E.Jonsson,A.Liebig,H.E.Wannberg,P.Nordblad,andB.Hjorvarsson.Finite-size effects in amorphous Fe90Zr10/Al75Zr25 mulyilayers. Physical. Review. B.85,214430-(1-7)(2012) 